\title{
Activators of Adenylate Cyclase and Cyclic AMP Prolong Calcium-Dependent Action Potentials of Mouse Sensory Neurons in Culture by Reducing a Voltage-Dependent Potassium Conductance
}

\author{
Debra S. Grega and Robert L. Macdonald \\ Department of Neurology, University of Michigan, Ann Arbor, Michigan 48109
}

\begin{abstract}
The effects of compounds that activate adenylate cyclase and of CAMP on calcium-dependent action potentials recorded from mouse dorsal root ganglion neurons were assessed. Application of compounds that stimulate the adenylate cyclase system (forskolin, cholera toxin, and prostaglandin $E_{1}$ ) increased action potential duration with an associated decrease in afterhyperpolarization. An adenylate cyclase inhibitor, $2^{\prime}, 5^{\prime}$-dideoxyadenosine, partially inhibited the responses to forskolin and cholera toxin. CAMP analogs mimicked the effect of forskolin, and the phosphodiesterase inhibitor theophylline enhanced the response to forskolin. Following intracellular injection of the potassium channel blocker cesium, the forskolin response was reduced. Forskolin did not significantly alter resting membrane potential or conductance. The action potential responses to forskolin were voltage dependent, being reduced when the membrane was held at less negative $(<-50 \mathrm{mV})$ potentials. The data suggest that activators of adenylate cyclase and cAMP prolong calcium-dependent action potentials by blocking a voltage-dependent potassium conductance that is responsible, in part, for action potential repolarization and that inactivates at membrane potentials less negative than $-50 \mathrm{mV}$.
\end{abstract}

Phosphorylation of ion channel proteins by cAMP-dependent protein kinase may be important in regulating neuronal membrane excitability (Kupfermann, 1980; Cohen, 1982; Nestler and Greengard, 1983). Intracellular cAMP concentrations are dependent upon the activity of adenylate cyclase, which catalyzes the conversion of ATP to CAMP, and by phosphodiesterase, which degrades cAMP to 5'-AMP (Ross and Gilman, 1980). Adenylate cyclase activity can be regulated by binding of neurotransmitters and hormones to stimulatory or inhibitory receptors, which are coupled to adenylate cyclase by at least 2 GTP binding regulatory proteins, $G_{s}$ and $G_{\mathrm{l}}$, respectively (see Gilman, 1984).

The actions of cAMP on membrane ion channels appear to differ among neurons (Levitan, 1986). These actions have been studied most completely in gastropod neurons, where cAMP

\footnotetext{
Received Jan. 24, 1986; revised Aug. 26, 1986; accepted Aug. 27, 1986.

We thank Mrs. Marjorie Mills for secretarial assistance. This work was supported by NIH NRSA NS 07231 to D.S.G. and United States Public Health Service Grants NS 19692 and NS 19613 to R.L.M.

Correspondence should be addressed to Robert L. Macdonald, M.D., Ph.D., University of Michigan, Neuroscience Laboratory Building, 1103 East Huron Street, Ann Arbor, MI 48104.

Copyright (C) 1987 Society for Neuroscience $0270-6474 / 87 / 030700-08 \$ 02.00 / 0$
}

increased potassium conductance (Pleurobranchea: Green and Gillette, 1983; Aplysia: Levitan and Adams, 1981; Pellman and Carpenter, 1981; Benson and Levitan, 1983; Ewald and Eckert, 1983; Helix: Deterre et al., 1981; Ewald et al., 1985), decreased potassium conductance (Helix: Swandulla and Lux, 1984; Aplysia: Siegelbaum et al., 1982; Kaczmarek and Strumwasser, 1984; Strong, 1984; Walsh and Byrne, 1984), and increased sodium or sodium/calcium conductance (gastropods: Connor and Hockberger, 1985; Helix: Doroshenko et al., 1984; Solntseva and Bezrukova, 1985; Aplysia: Pellman and Carpenter, 1981). In Aplysia sensory neurons, 5-HT has been shown to regulate a potassium channel by cAMP-dependent protein phosphorylation (Camardo et al., 1983).

In vertebrate neurons and cells, modification of intracellular cAMP has been shown also to have varied effects (Kupfermann, 1980; Levitan, 1986). In Xenopus, cAMP increased both potassium and sodium conductances (oocytes: Van Renterghem et al., 1985; node of Ranvier: Seelig and Kendig, 1982). In cardiac cells, calcium current was enhanced by an epinephrinesensitive adenylate cyclase (Reuter, 1983). In mouse neuroblastoma cells, adenosine and prostaglandin $E_{1}$, which are known to increase cAMP levels, potentiated an ACh-induced hyperpolarization (Tsunoo and Narahashi, 1984). In a fibroblast cell line, prostaglandin $E_{1}$ increased intracellular cAMP(Kelly et al., 1974). This prostaglandin $E_{1}$-stimulated increase in cAMP was partially inhibited by $2^{\prime}, 5^{\prime}$-dideoxyadenosine (Bruns, 1980). In mutant hamster ovary cells, gap junctional permeability appears to be regulated by a cAMP-dependent protein kinase (Wiener and Loewenstein, 1983). Dunlap (1985) reported that forskolin, an adenylate cyclase activator (Seamon and Daly, 1981), decreased voltage-dependent potassium current in cultured chick sensory neurons. Purified sodium ion channels from rat brain (Costa et al., 1982) and ACh receptor/channel from Torpedo (Huganir and Greengard, 1983) have been shown to be phosphorylated by cAMP protein kinase in vitro. The phosphorylation does alter an appropriate ionic flux $\left(\mathrm{Na}^{+}\right.$: Costa and Catterall, 1984), although the physiological correlate to normal channel functioning has yet to be established.

$W_{c}$ have investigated alterations in the duration and configuration of action potentials recorded from mouse dorsal root ganglion (DRG) neurons in culture produced by compounds that stimulate the hormone-sensitive adenylate cyclase system. We report that these compounds increased calcium-dependent action potential duration and suggest that the action potential prolongation is due to reduction of a voltage-dependent potassium conductance. These results have been presented previously in abstract form (Grega and Macdonald, 1985). 


\section{Materials and Methods}

Neuronal cultures were prepared, as previously described (Ransom et al., 1977), by dissecting spinal cords with attached DRG from 12- to 14-d-old fetal mice, mechanically dissociating the tissue, and plating the resultant cell suspension on $35 \mathrm{~mm}$ collagen-coated plates at a density of $1 / 4$ spinal cord per plate. Initial culture medium consisted of $73 \%$ Eagle's minimum essential medium, $\mathrm{NaHCO}_{3}(1.5 \mathrm{~g} /$ liter $)$, glucose $(5.5$ $\mathrm{g} /$ liter), $9 \%$ horse serum, $9 \%$ fetal calf serum (Gibco), and $9 \%$ NuSerum (Collaborative Research). Two days after plating the growth medium was modified to $90 \%$ Eagle's minimum essential medium with $\mathrm{NaHCO}_{3}$ and glucose, $5 \%$ horse serum, and 5\% NuSerum. Five days after initial plating, $5^{\prime}$ fluoro-2'-deoxyuridine and uridine were added to the cultures to inhibit the division of non-neuronal background cells. All culture media were changed 3 times weekly and contained nerve growth factor at a concentration of $5 \mathrm{ng} / \mathrm{ml}$ to promote survival and growth of DRG neurons. Three- to six-week-old cultures were used in electrophysiological experiments.

Intracellular recording. For electrophysiological study, cultures were placed on the heated stage $\left(33^{\circ} \mathrm{C}\right)$ of an inverted phase-contrast microscope, which allowed neuronal impalement by a recording micropipette under visual observation. DRG neurons were identified by their distinctive morphology (Ransom et al., 1977). Intracellular recordings were obtained from DRG neurons using high-resistance (20-50 M 2 ) micropipettes filled with $4 \mathrm{M}$ potassium acetate (KAc) or $3 \mathrm{M}$ cesium acetate (CsAc), a potassium channel blocker. A modified bridge circuit allowed "simultaneous" current passage and voltage recording using single micropipettes. During impalement with a CsAc micropipette, the membrane potential decreased to 0 to $-20 \mathrm{mV}$, input conductance decreased, action potential duration increased to 100 to $600 \mathrm{msec}$, and the afterhyperpolarization was abolished, consistent with substantial reduction of potassium conductance following intracellular cesium injection.

For recording, neurons were bathed in a Tris-buffered saline (TBS)

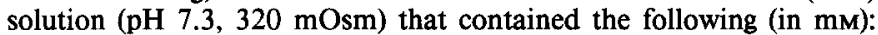
$\mathrm{NaCl}, 142.5 ; \mathrm{KCl}, 5.3 ; \mathrm{MgCl}_{2}, 0.8 ; \mathrm{CaCl}_{2}, 5.0 ;$ glucose, 5.6; Tris, 13.0. In most experiments, tetraethylammonium chloride (TEA), $5.0 \mathrm{~mm}$ was added to the TBS medium (TBS-TEA). TEA, which decreases some voltage-dependent potassium conductances (Armstrong and Binstock, 1965), was used to increase the duration of the calcium component of DRG neuron action potentials. Evaporation with associated increased osmolality was prevented by placing a thin coat of mineral oil over the recording medium.

Following impalement of DRG neurons and stabilization of membrane potential, brief $(500 \mu \mathrm{sec})$ depolarizing current pulses were applied to evoke action potentials at a frequency of $4 / \mathrm{min}$. The action potentials had an early ( $<3 \mathrm{msec}$ ) sodium-dependent component and a long calcium-dependent component. Action potentials were evoked before and after drug application and were recorded on photographic film. Action potential duration was measured from the stimulus onset to the time when the original membrane potential was restored. Afterhyperpolarizations were not included in the duration measurement. Membrane potential was monitored on a polygraph.

Drugs. Drug dilutions in recording buffer were made immediately prior to use. Forskolin (Calbiochem, La Jolla, CA) (10 mM) was dissolved in dimethyl sulfoxide (DMSO), aliquoted, and frozen. Application of a DMSO control dilution $(1: 100)$ produced less than a $4 \%$ change in action potential duration. Cholera toxin, prostaglandin $\mathrm{E}_{1}$, theophylline, and cAMP analogs were obtained from Sigma Chemical Company (St. Louis, MO). Cholera toxin was reconstituted at $1 \mathrm{mg} / \mathrm{ml}$ for a stock solution and kept refrigerated. Prostaglandin $E_{1}(10 \mathrm{~mm})$ was dissolved in DMSO, aliquoted, and frozen. Solutions of $2^{\prime}, 5^{\prime}$-dideoxyadenosine, obtained courtesy of Dr. Fred Bruns (Warner-Lambert, Ann Arbor, MI), and of theophylline were made immediately before use. The AMP analogs, $N^{6},-2^{\prime}-O$-dibutyryladenosine $3^{\prime}: 5^{\prime}$-cyclic monophosphate (DbcAMP), and 2'-O-monobutyryl-8-bromoadenosine $3^{\prime}: 5^{\prime}$ cyclic monophosphate (8BcAMP), and 8-(4-chlorophenylthio)-adenosine 3':5'-cyclic monophosphate (CPTcAMP) were made fresh and kept as frozen stocks of $10 \mathrm{~mm}$ in distilled water.

Drug application by pressure ejection. Drug solutions were applied by pressure ejection $(0.8 \mathrm{psi})$ from micropipettes (with tip diameters of $2-5 \mu \mathrm{m})$ prior to evoking an action potentiai. Drug-containing pipettes were positioned to within $5 \mu \mathrm{m}$ of the cell soma immediately prior to pressure application and were then removed from the cell environment. In TBS medium, forskolin and cholera toxin were ejected for $5 \mathrm{sec}$ at $7 \mathrm{sec}$ prior to evoking an action potential. In TBS-TEA medium, the duration of drug application varied with the different substances. Forskolin was delivered for $1 \mathrm{sec}$ ejection at $5 \mathrm{sec}$ prior to evoking an action potential; cholera toxin for an ejection of $3 \mathrm{sec}$ at $6 \mathrm{sec}$ prior to evoking an action potential; prostaglandin $E_{1}, 2$ sec ejection at $6 \mathrm{sec}$ prior to action potential; and the cyclic nucleotides, $4 \mathrm{sec}$ at $7 \mathrm{sec}$ prior to action potential. The delivery of drug solution was optimized to obtain a consistent response while minimizing mechanical artifacts associated with delivery during an action potential. Three to $4 \mathrm{~min}$ were allowed to elapse between drug applications.

\section{Results}

DRG neuron action potentials were previously determined to have 2 components: An initial sodium-dependent component that was only partially blocked by TTX as well as a slower calcium-dependent component, which together resulted in action potential durations of 3-4 msec (Heyer and Macdonald, 1982). To broaden the action potential and enhance the calcium component of the action potentials, a recording medium containing the potassium channel blocker TEA ( $5 \mathrm{~mm}$ ) was used (TBS-TEA medium). At $5 \mathrm{~mm}$, TEA blocks a portion of the delayed rectifier current (Armstrong and Binstock, 1965; D. S. Grega and R. L. Macdonald, unpublished observations) but does not modify inactivating or noninactivating voltage-dependent calcium currents (R. A. Gross and R. L. Macdonald, unpublished observations) in DRG neurons. In most experiments, the majority of the action potential plateau was dependent upon calcium, as indicated by blockade of the plateau by cadmium, a calcium channel blocker. The presence of 15-25 $\mathrm{mV}$ afterhyperpolarizations following action potentials indicated substantial potassium conductance even in the presence of TEA.

\section{Forskolin, cholera toxin, and prostaglandin $E_{I}$ increased action potential duration}

Forskolin directly activates adenylate cyclase (Seamon and Daly, 1981), while cholera toxin indirectly activates adenylate cyclase by ADP ribosylation of $\mathrm{G}_{\mathrm{S}}$ (Limbird, 1981). Forskolin $(10 \mu \mathrm{M})$ and cholera toxin $(10 \mu \mathrm{g} / \mathrm{ml})$ increased action potential duration 30.5 and $21.2 \%(n=9)$, respectively, when applied to neurons bathed in TBS medium. The increase was maximal on the first action potential after the application and returned to the control value within $1 \mathrm{~min}$. However, action potential duration in TBS medium was brief (3-4 msec) and the calcium-dependent component small $(<1 \mathrm{msec})$. To facilitate quantitation of alterations in the duration of calcium-dependent component of the action potential, TEA was included in the recording medium to increase action potential duration (see Materials and Methods). In TBS-TEA medium, forskolin $(10 \mu \mathrm{M})$ initially increased action potential duration with a decrease in the amplitude of the afterhyperpolarization (Fig. $1 A$ ). The action potential prolongation, which was maximal on the first action potential after the $1 \mathrm{sec}$ application of forskolin, lasted for approximately 30 $60 \mathrm{sec}$. Subsequent to the prolongation, the action potential duration passed through the original duration and shortened, usually with an increase in afterhyperpolarization (Fig. 1 $A$ ). The original action potential duration was regaincd within 2 min after forskolin application. The magnitude of the forskolin effect was dose dependent from 1 to $100 \mu \mathrm{M}$, with larger increases and subsequent decreases in action potential duration (Fig. 2) and decreases in afterhyperpolarization (Table 1). Cholera toxin (10 $\mu \mathrm{g} / \mathrm{ml}$ ) mimicked the forskolin effect of action potential prolongation and shortening (Fig. $1 B$ ). The action potential prolongation could be sustained with longer (seconds to minutes) application of forskolin or cholera toxin. Prostaglandin $E_{1}(1 \mathrm{~mm})$, 
A. FOR

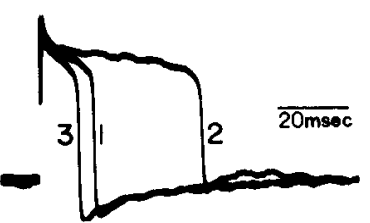

B. $C T$

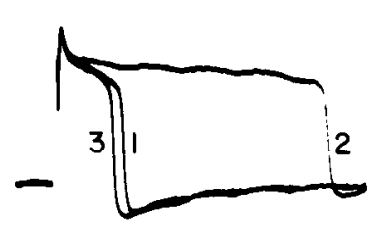

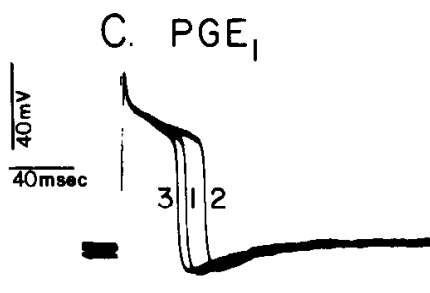

Figure 1. Stimulation of the adenylate cyclase system increased action potential duration. Forskolin $(F O R, 10 \mu \mathrm{M})$, cholera toxin $(C T, 10 \mu \mathrm{g} / \mathrm{ml})$, and prostaglandin $\mathrm{E}_{1}\left(P G E_{1}, 1 \mathrm{~mm}\right)$ were applied after evoking a control action potential $(1)$ and prior to evoking a second action potential (2). Action potential duration was increased by all 3 compounds. The second action potential evoked following drug application was reduced (action potential 1 vs 3 ). Note the decrease in the afterhyperpolarization with action potential 2 and the subsequent increase in afterhyperpolarization with action potential 3 .

which activates adenylate cyclase via a stimulatory membrane surface receptor (see Ross and Gilman, 1980), also produced action potential prolongation and subsequent shortening but was less potent and less efficacious than forskolin or cholera toxin (Fig. 1C).

The prolongation of DRG action potentials by forskolin was not associated with significant changes in membrane potential or membrane conductance. Forskolin $(10 \mu \mathrm{M})$ was applied to DRG neurons prior to evoking an action potential, and action potential duration increased (Fig. $3 \mathrm{Al}$ ); membrane potential was usually unaltered during or following forskolin application (Fig. $3 A 2)$. When membrane potential was held at $-60 \mathrm{mV}$ and no action potentials were evoked, application of forskolin produced a slight increase in resting membrane conductance $(5.4 \%$ increase; $n=6$; Fig. $3 B$ ). Occasionally ( 2 of 6 ) a slight depolarization was associated with the change in conductance. Recovery to the control value was achieved within several minutes.

\section{Increased action potential duration partially blocked by 2',5'-dideoxyadenosine}

The adenylate cyclase inhibitor $2^{\prime}, 5^{\prime}$-dideoxyadenosine was used to assess the effect of reducing adenylate cyclase activity. Forskolin (10 $\mu \mathrm{M}$; Fig. $4 A I)$ and cholera toxin $(10 \mu \mathrm{g} / \mathrm{ml}$; Fig. $4 A 2)$

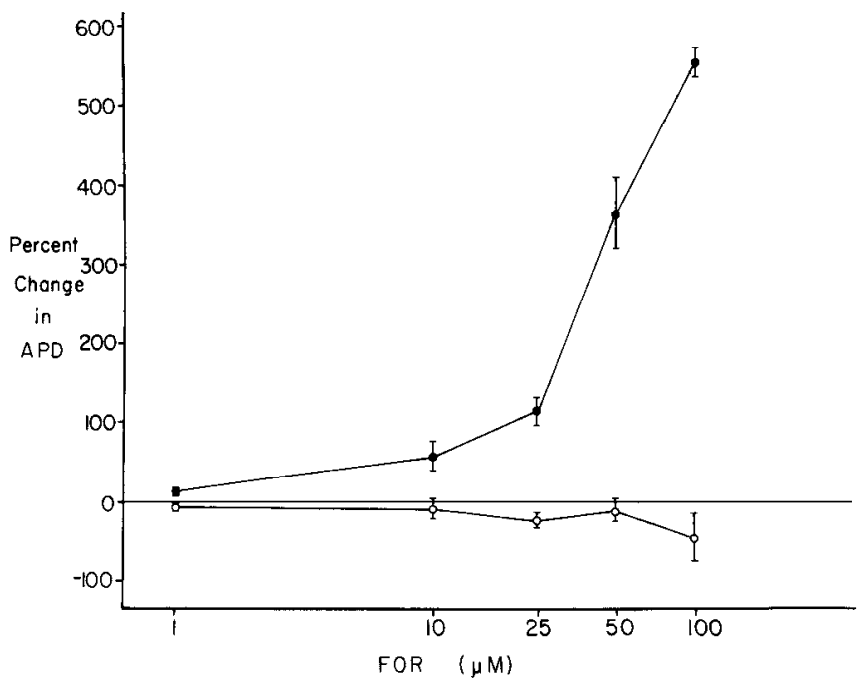

Figure 2. Forskolin (FOR) effect on action potential duration was concentration dependent. Filled circles represent action potential duration prolongation immediately after a $1 \mathrm{sec}$ FOR application; empty circles indicate the subsequent shortening in action potential duration observed following the initial prolongation. The values are the average of the responses of 8 neurons \pm SEM. were applied to DRG neurons, and action potential durations increased. Often, action potential duration gradually increased over time, as can be seen by comparing control (1) action potential durations in Figure $4, A$ and $B$. The percentage changes from control duration were compared. $2^{\prime}, 5^{\prime}$-Dideoxyadenosine $(100 \mu \mathrm{M})$ was applied via a large-bore micropipette $1 \mathrm{~min}$ prior to and during the application of forskolin and cholera toxin. $2^{\prime}, 5^{\prime}$-Dideoxyadenosine partially inhibited both forskolin (Fig. $4 B 1$ ) and cholera toxin (Fig. 4B2) effects. Several minutes after removal of 2',5'-dideoxyadenosine, forskolin (Fig. 4CI) and cholera toxin (Fig. 4C2) were reapplied, and the percentage increase in action potential duration was comparable to that observed before $2^{\prime}, 5^{\prime}$-dideoxyadenosine application. 2',5'-Dideoxyadenosine inhibited action potential prolongation produced both by forskolin (by $38 \% ; n=6$ ) and by cholera toxin (by $42.3 \% ; n=4$ ), with mixed results (enhancement and inhibition) on the subsequent shortening. The blockade of forskolin and cholera toxin actions by $2^{\prime}, 5^{\prime}$-dideoxyadenosine was always reversible.

\section{CAMP analogs prolonged action potential duration}

Application of analogs of cAMP (8BcAMP, DbcAMP, and CPTcAMP) yielded mixed results. 8BcAMP (1 mM) was the most effective of the 3 analogs with a $16.8 \%$ increase in action potential duration and a subsequent $9.2 \%$ decrease in action potential duration ( $n=7$; Fig. $5 A$ ). DbcAMP resulted in $8.2 \%$ $(10 \mu \mathrm{M}, n=4)$ and $11.1 \%(100 \mu \mathrm{M}, n=4)$ action potential duration increases, but inconsistent results at $1 \mathrm{mM}$. Application of CPTcAMP ( $1 \mathrm{mM}$ ) also gave inconsistent effects on action potential duration.

\section{Theophylline prolonged the action of forskolin on action potential duration}

Theophylline, a phosphodiesterase inhibitor, was used to block degradation of cAMP produced following forskolin activation

\begin{tabular}{|c|c|c|}
\hline $\begin{array}{l}\text { Forskolin } \\
\text { concentration } \\
(\mu \mathrm{M})\end{array}$ & $\begin{array}{l}\text { Afterhyperpolarization } \\
\text { reduction } \\
\text { (\%) }\end{array}$ & $N$ \\
\hline 100 & 53.1 & 7 \\
\hline 50 & 53.4 & 7 \\
\hline 25 & 63.8 & 9 \\
\hline 10 & 5.3 & 8 \\
\hline 1 & 10.4 & 5 \\
\hline
\end{tabular}



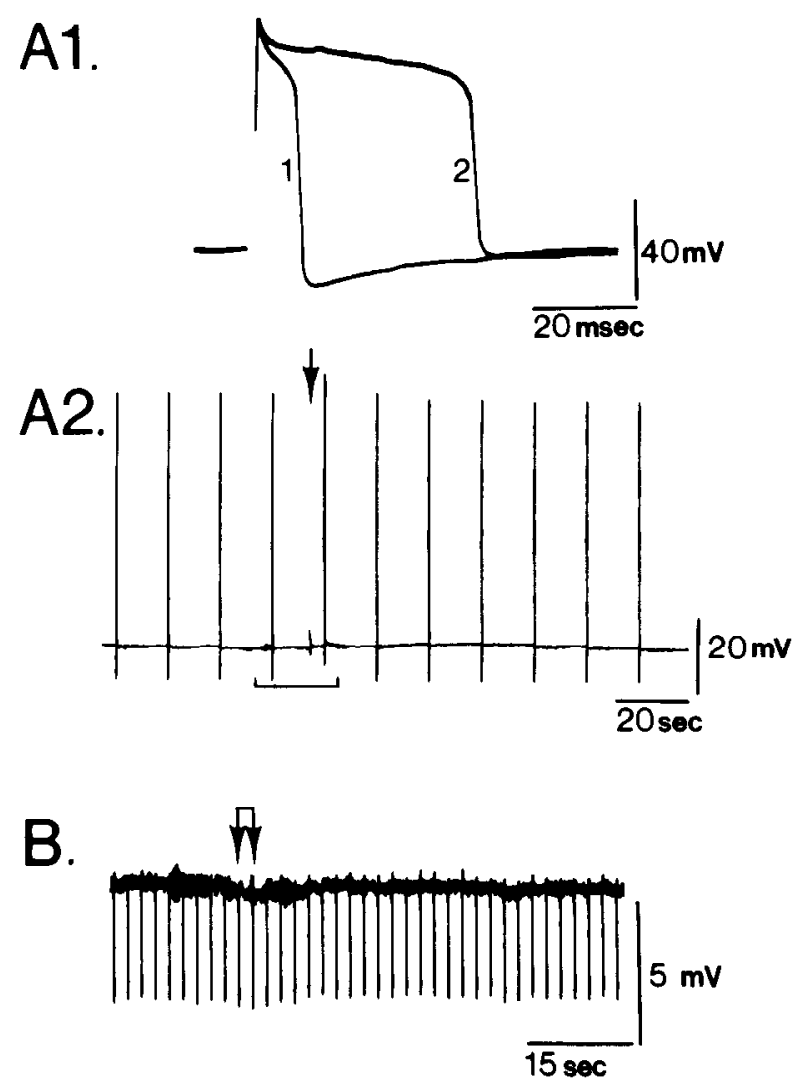

Figure 3. Application of forskolin (FOR) had little effect on resting membrane potential or conductance. FOR $(10 \mu \mathrm{M})$ application (arrow) immediately prior to evoking an action potential elicited a large increase in action potential duration $(A I)$ with the associated decrease in afterhyperpolarization (A2, bracket). FOR application (between the arrows) to the same neuron produced little change in resting membrane conductance $(B)$ as assessed with brief hyperpolarizing pulses.

of adenylate cyclase. Initially, theophylline ( $1 \mathrm{mM}$ ) was applied from a micropipette at the time of forskolin $(10 \mu \mathrm{M})$ application. This approach, however, gave inconsistent results. Therefore, theophylline was bath applied (final concentration, $1 \mathrm{~mm}$ ) midway through an experiment. Some neurons $(n=3)$ were impaled prior to (Fig. $5 B 1$ ) and after theophylline (Fig. $5 B 2$ ) was added to the bath and exhibited $170.9 \%$ enhancement of the forskolin response after bathing in theophylline. Recordings were also obtained from DRG neurons bathed in TBS-TEA medium with and without theophylline. Forskolin $(10 \mu \mathrm{M})$ produced a $131.9 \%$ increase in action potential duration $(n=6)$ in medium containing theophylline compared to a $56.1 \%$ increase in action potential duration $(n=8)$ in control medium without theophylline.

\section{Blockade of potassium conductance by intracellular cesium injection eliminated action potential prolongation by forskolin}

Forskolin decreased the amplitude of the afterhyperpolarization following action potentials (Fig. 1, Table 1) and prolonged action potential duration, suggesting that forskolin reduced a potassium conductance. To confirm this, DRG neurons $(n=5)$ were impaled with CsAc micropipettes and time was permitted for diffusion of the cesium into the cell. Substantial block of potassium channels was produced, as indicated by a decrease in the membrane potential, a large reduction or loss of the afterhyperpolarization and an increase in the action potential duration to more than $200 \mathrm{msec}$. Two neurons were initially impaled with KAc-filled micropipettes and responded to forskolin $(10 \mu \mathrm{M}$ ) application with $184 \%$ (Fig. $6 A 1$ ) and $60 \%$ (Fig. $6 B 1$ ) increases in action potential duration. After reimpalement with CsAc-filled micropipettes, increases of only $1.8 \%$ (Fig. 6A2) and $1.1 \%$ (Fig. $6 B 2$ ) were produced. Three other neurons were impaled only with CsAc-filled micropipettes. Forskolin produced a $5.9 \%$ increase in action potential duration compared to an average $50 \%$ increase in action potential duration from DRGs impaled with KAc-filled micropipettes (Fig. 2).

\section{Forskolin-induced increase in action potential duration was voltage dependent}

The increase in action potential duration produced by forskolin was voltage-dependent in TBS medium. Following membrane depolarization to -20 to $-40 \mathrm{mV}$, forskolin $(100 \mu \mathrm{M})$ prolongation of action potentials was reduced by $73.6 \%(n=5)$. Similar results were obtained in TEA-TBS medium. Application of forskolin increased action potential duration and reduced action potential afterhyperpolarization at membrane potentials greater than $-50 \mathrm{mV}$ (Fig. 7A). Following membrane depolarization to less than $-50 \mathrm{mV}$, action potential duration increased over
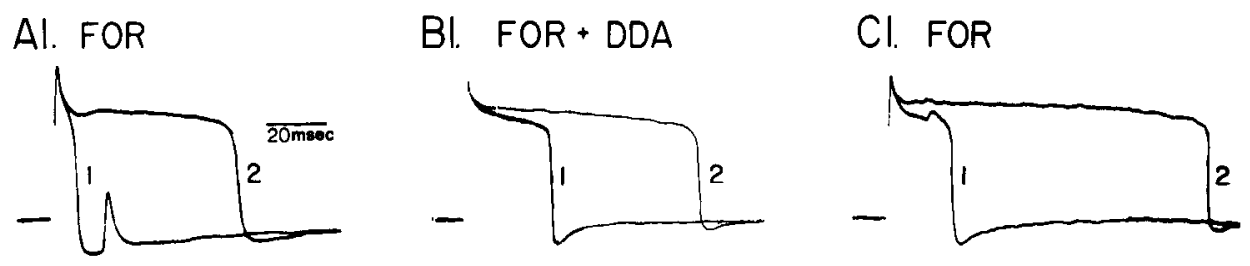
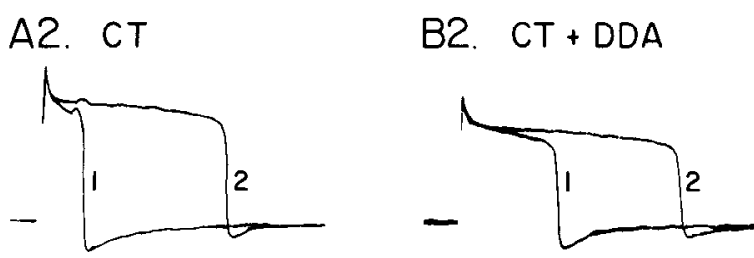

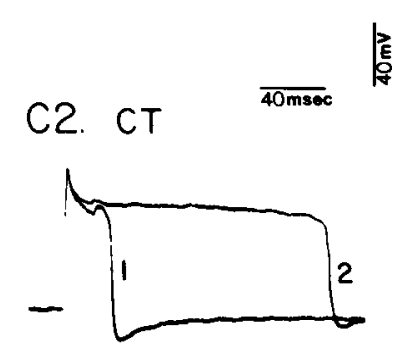

Figure 4. Adenylate cyclase inhibitor $(D D A)$ partially inhibited action potential prolongation by both forskolin $(F O R, 10 \mu \mathrm{M})$ and cholera toxin $(C T$, $10 \mu \mathrm{g} / \mathrm{ml})$. DDA $(100 \mu \mathrm{M})$ decreased the FOR prolongation by $42.3 \%$ and the CT effect by $56.1 \%$. Following removal of DDA, the responses to FOR and CT returned to control or greater than control values. 


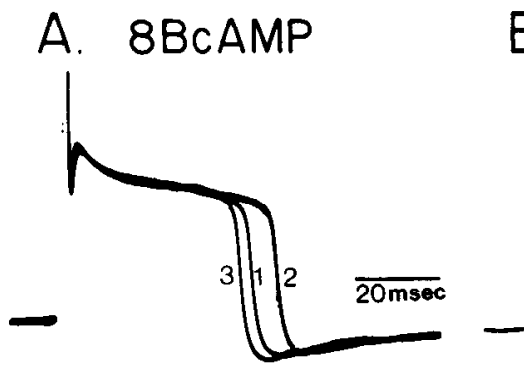

Bl. FOR B2. FOR + Theo
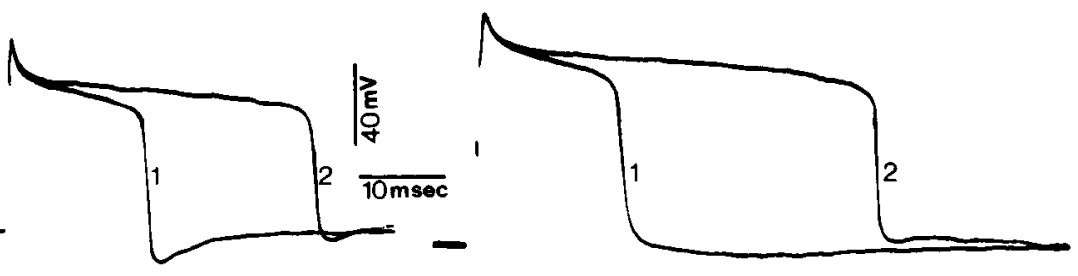

Figure 5. cAMP and cAMP analogs increased action potential duration. With the application of exogenous cAMP, $1 \mathrm{mM} 8 \mathrm{BcAMP}(A)$ or with the addition of theophylline (Theo; final concentration, $1 \mathrm{mM}$ ) to the bathing medium with FOR (10 $\mu \mathrm{M})$ application $(B 2)$ action potential duration was increased.

several minutes and action potential afterhyperpolarization was reduced or abolished (Fig. $7 B$ ). Following stabilization of the increased action potential duration, the forskolin effect was diminished (Fig. 7B). Following membrane repolarization to greater than $-50 \mathrm{mV}$, action potential duration was reduced to the control duration, and the action potential afterhyperpolarization reappeared. After action potential duration had stabilized, the forskolin effect returned and was comparable to that produced at the original membrane potential (Fig. $7 \mathrm{C}$ ). The magnitude of the response to forskolin was relatively constant when action potentials were evoked from membrane potentials more negative than about $-50 \mathrm{mV}$ (Figs. $7, A, C ; 8$ ). However, with further membrane depolarization, the effect of forskolin on action potential duration was substantially reduced (Figs. $7 B, 8$ ). At membrane potentials less negative than $-40 \mathrm{mV}$, only small $(<20 \%)$ action potential prolongations were produced (Fig. 8). Over the range of about -50 to $-40 \mathrm{mV}$, the forskolin effect was steeply voltage dependent (Fig. 8).

\section{Discussion}

Application of compounds that stimulate adenylate cyclase or application of cAMP analogs increased calcium-dependent ac- tion potential duration of mouse DRG neurons in cell culture. The potency of compounds proven to affect the adenylate cyclase system (stimulators such as forskolin, cholera toxin, and prostaglandin $\mathrm{E}_{1}$, as well as the inhibitor 2',5'-dideoxyadenosine), in addition to the effect of theophylline, suggested that the enhancement of action potential duration was due to an increase in intracellular CAMP and thus to activation of CAMPdependent protein kinase. The effectiveness of prostaglandin $E_{1}$ and cholera toxin suggest that excitatory hormone receptors are present on DRG neurons which are coupled to adenylate cyclase by $\mathrm{G}_{\mathrm{s}}$. However, the observations presented in this paper do not unequivocally establish the presence of a hormone-regulated adenylate cyclase/cAMP-dependent protein kinase system. The effects of all stimulators; including cholera toxin, were rapid in onset $(<15)$ sec and were rapidly reversible. Furthermore, an increase in cAMP concentrations produced by the stimulatory compounds has not been demonstrated to precede or to coincide with the electrophysiological effects. Thus, it is possible that the electrophysiological effects described here are unrelated to actions on the adenylate cyclase system.

The prolongation of action potential duration produced by forskolin, cholera toxin, and prostaglandin $E_{1}$ was most likely

$$
\text { KAc }
$$

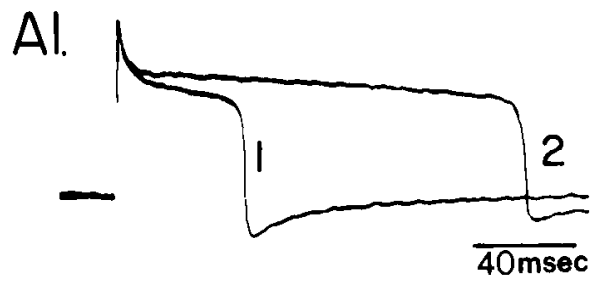

Figure 6. Intracellular injection of cesium blocked action potential prolongation by forskolin. When impaled with a KAc micropipette, forskolin $(10 \mu \mathrm{M})$ prolonged action potential duration by $184 \%(A I)$ or by $60 \%(B I)$. Following reimpalement of the same neuron with a CsAc micropipette, the response to forskolin was reduced to $1.8 \%(A 2)$ and $1.1 \%(B 2)$.

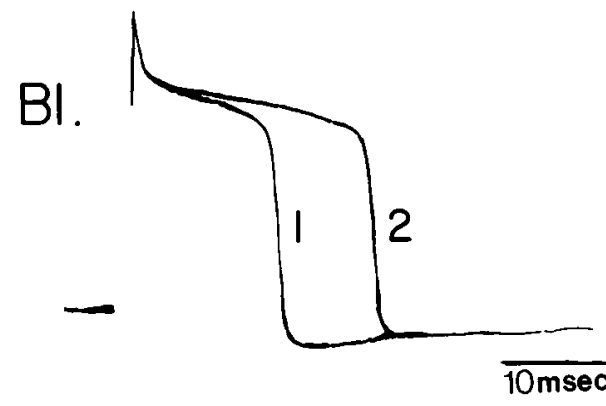

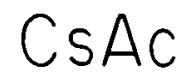

A2.

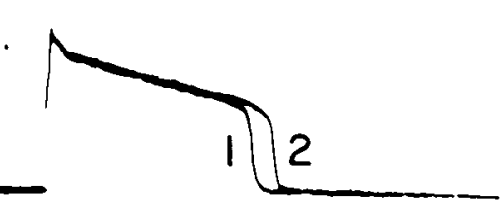

|

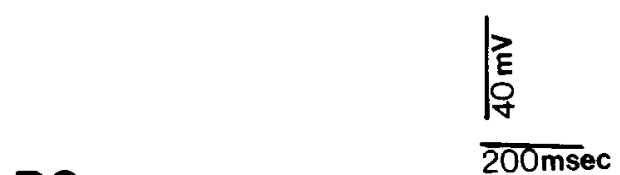

B2.

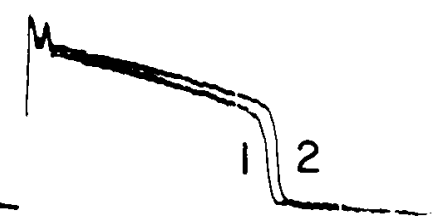




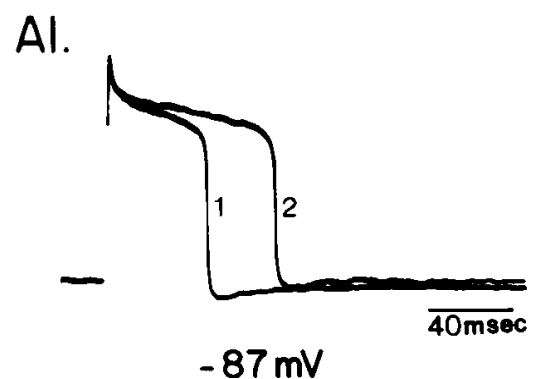

$\mathrm{BI}$

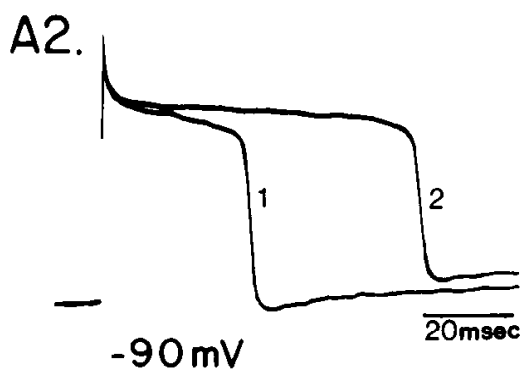

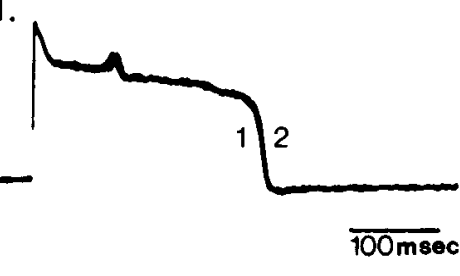

$-40 \mathrm{mV}$
$\mathrm{Cl}$.

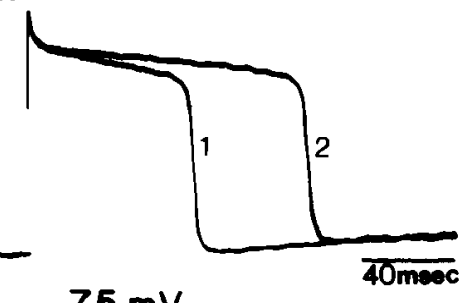

PE

Figure 7. Action potential duration increase by forskolin was dependent upon membrane potential. Application of $10 \mu \mathrm{M}$ forskolin produced an increase in action potential duration when membrane potential was held at greater than $-70 \mathrm{mV}(A 1, A 2)$. Forskolin was ineffective $(B 1)$ or less ineffective $(B 2)$ when the membrane potential was held at less negative potentials $(<-50 \mathrm{mV})$. The response to forskolin returned when the membrane potentials were returned to more negative potentials $(C 1, C 2)$.

due to a decrease in a voltage- and/or calcium-dependent potassium conductance and/or an increase in a voltage-dependent calcium conductance. It is difficult to be certain which conductances were altered by forskolin, cholera toxin, and prostaglan$\operatorname{din} E_{1}$, without using the voltage-clamp technique. However, several observations suggest that the stimulatory compounds increased action potential duration, at least in part, by reducing a voltage-dependent potassium conductance. First, action potential prolongation was accompanied by reduction of the potassium-dependent afterhyperpolarization. Second, following membrane depolarization and reduction of the potassium-de- pendent afterhyperpolarization, the effect of forskolin was reduced or abolished. Third, injection of the potassium conductance blocker, cesium ion, abolished the effect of forskolin on action potential duration. In addition, Dunlap (1985) and D. S. Grega, M. A. Werz, and R. L. Macdonald (unpublished observations) have demonstrated that forskolin reduces a voltagedependent outward potassium current in DRG neurons.

Several observations suggest that the forskolin, cholera toxin, and prostaglandin $E_{1}$ did not have a quantitatively major effect on calcium channels to prolong action potential duration. First, the effect of forskolin was blocked by intracellular cesium in-

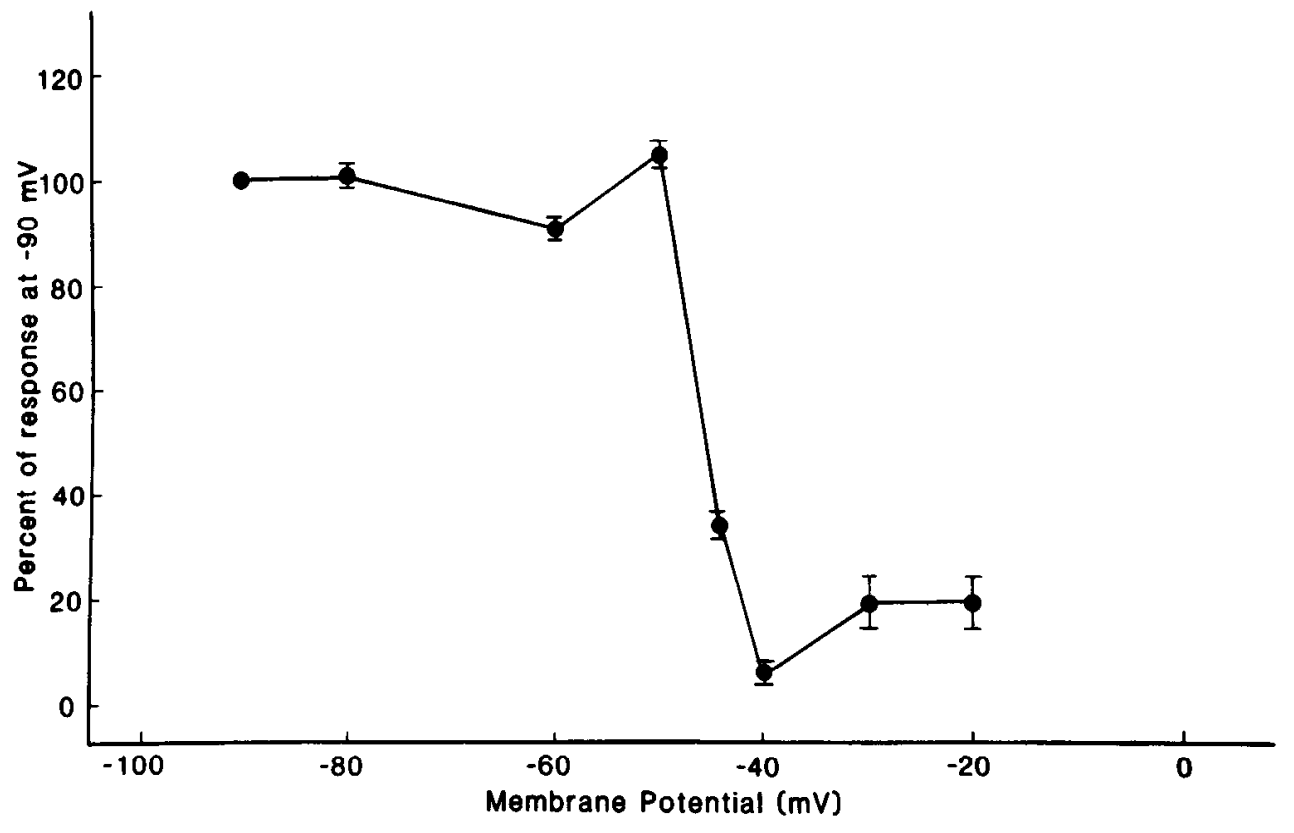

Figure 8. Magnitude of the forskolin effect was voltage dependent. The magnitude of action potential duration prolongation as a percentage of the response at $-90 \mathrm{mV}$ is plotted as a function of the membrane potential. Forskolin was applied following stabilization of action potential duration at each membrane potential. There was a decrease in forskolin response at potentials less than $-50 \mathrm{mV}$; the decreased response was reversible at more negative membrane potentials. The means \pm SEM are plotted for 6 neurons in response to $10 \mu \mathrm{M}$ forskolin. 
jection without altering the potential from which action potentials were evoked. Second, following membrane depolarization to less than $-50 \mathrm{mV}$, calcium conductance was not fully inactivated (Nowycky et al., 1985) and calcium-dependent action potentials with less than maximal duration (i.e., $<1000 \mathrm{msec}$ ) were evoked. However, the forskolin effect was reduced or abolished at these holding potentials. Third, minimal or no elevation of the action potential plateau was produced by forskolin following intracellular cesium injection or membrane depolarization. Thus, forskolin had no effect under conditions where potassium conductance was blocked but submaximally long calcium-dependent action potentials could be evoked over a range of holding potentials. These observations suggest that the predominant effect of forskolin was not on calcium conductance.

While these observations do not exclude an effect of forskolin on calcium conductance, they suggest that forskolin does decrease a potassium conductance and that the action potential is prolonged, in large part, due to reduction of potassium current. Confirmation of the identity of the potassium conductance remains to be determined by voltage-clamp experiments. Based on previously published studies (for review, see Levitan, 1986), it is likely that multiple types of ion channels are modified by cAMP, and the effect on action potential duration in any given cell type would depend on the relative effect on the different types of channels.

The cAMP analogs did not demonstrate as definitive effects as forskolin, possibly because of difficulty crossing the cell membrane. The fact that prostaglandin $E_{1}$ was less potent than forskolin or cholera toxin might be due to less than completely effective coupling between prostaglandin $E_{1}$ receptors and $G_{s}$ proteins. In rabbit superior cervical ganglion neurons, prostaglandin $\mathrm{E}_{1}$ inhibited calcium-dependent potentials (Mo et al., 1985). The basis for the difference between these studies is unclear. While DRG neurons have a stimulatory prostaglandin $\mathrm{E}_{1}$ receptor, it is unclear whether adenosine (Macdonald et al., 1986), catecholamine (Dunlap and Fischbach, 1978), or opiate (Werz and Macdonald, 1983, 1985) effects on DRG neuron calcium-dependent action potential duration are mediated by receptors coupled to adenylate cyclase.

The delayed shortening of action potential duration following forskolin application may be due to activation of a calciumdependent potassium conductance or reduction of calcium conductance. The calcium-dependent potassium conductance could be stimulated by the increase in intracellular calcium produced by prolongation of the calcium-dependent action potential.

cAMP-dependent modulation of potassium channels is well documented in invertebrate neurons. In Aplysia abdominal ganglion sensory neurons, the transmitter 5-HT elevated cAMP, which, via a protein kinase, decreased a potassium conductance. The actual closure of single potassium channels by 5-HT and cAMP is well established (Siegelbaum et al., 1982). The resulting increase in action potential duration underlies the behavioral sensitization of the gill-withdrawal reflex (see Kandel and Schwartz, 1982). In Aplysia bag cells, elevated levels of cAMP depress both the delayed rectifying potassium and early transient potassium currents (Kaczmarek and Strumwasser, 1984; Strong, 1984). In Helix, cAMP decreased potassium permeability (Swandulla and Lux, 1984), while in Pleurobranchaea, cAMP appeared to depress a calcium-activated potassium current (Gillette et al., 1982).

In vertebrate cells, elevated adenylate cyclase activity/cAMP levels tend to increase conductances: Calcium conductance in cardiac tissue (Reuter, 1983), gap junctional permeability in ovary cells (Wiener and Loewenstein, 1983), and induced hyperpolarization in neuroblastoma cells (Tsunoo and Narahashi, 1984). However, for chick sensory neurons, Dunlap (1985) reported that forskolin decreased a voltage-sensitive potassium conductance. Forskolin was effective over the same concentration range (1-100 $\mu \mathrm{M})$ in chick (Dunlap, 1985) and mouse DRG neurons. Thus, there appears to be a similarity in the response of sensory neurons, regardless of phyletic affiliation, to increased adenylate cyclase activity, i.e., decreasing a potassium conductance. Further investigation utilizing whole-cell and isolated patch-clamp techniques should provide more information concerning the similarities and differences between the adenylate cyclase systems of vertebrate and invertebrate neurons.

\section{References}

Armstrong, C. M., and L. Binstock (1965) Anomalous rectification in the squid giant axon injected with tetraethylammonium chloride. J. Gen. Physiol. 48: 859-872.

Benson, J. A., and I. B. Levitan (1983) Serotonin increases an anomalously rectifying $\mathrm{K}^{+}$current in Aplysia neurons R15. Proc. Natl. Acad. Sci. USA 80: 3522-3525.

Bruns, R. F. (1980) Adenosine receptor activation in human fibroblasts: Nucleoside agonists and antagonists. Can. J. Physiol. Pharmacol. 58: 673-691.

Camardo, J. S., M. J. Shuster, S. A. Siegelbaum, and E. R. Kandel (1983) Modulation of a specific potassium channel in sensory neurons of Aplysia by serotonin and cAMP-dependent protein phosphorylation. Cold Spring Harbor Symp. Quant. Biol. 48: 213-220.

Cohen, P. (1982) The role of protein phosphorylation in neural and hormonal control of cellular activity. Nature 296: 613-620.

Connor, J. A., and P. Hockberger (1985) Calcium and cAMP: Second messengers in gastropod neurons. In Model Neural Networks and Behavior, A. I. Selverston, ed., pp. 437-460, Plenum, New York.

Costa, M. R. C., and W. A. Catterall (1984) Cyclic AMP-dependent phosphorylation of the $\alpha$ subunit of the sodium channel in synaptic nerve ending particles. J. Biol. Chem. 259: 8210-8218.

Costa, M. R. C., J. E. Casnellie, and W. A. Catterall (1982) Cyclic AMP-dependent phosphorylation of the $\alpha$ subunit of the sodium channel by cAMP-dependent protein kinase. J. Biol. Chem. 257: 79187921.

Deterre, P., D. Paupardin-Tritsch, J. Bockaert, and H. M. Gerschenfield (1981) Role of cyclic AMP in a serotonin-evoked slow inward current in snail neurones. Nature 290: 783-785.

Doroshenko, P. A., P. G. Kostyuk, A. E. Martynyuk, M. D. Kursky, and Z. D. Yorobetz (1984) Intracellular protein kinase and calcium inward currents in perfused neurones of the snail Helix pomatia. Neuroscience 11: 263-267.

Dunlap, K. (1985) Forskolin prolongs action potential duration and blocks potassium current in embryonic chick sensory neurons. Pfluegers Arch. 403: 170-174.

Dunlap, K., and G. D. Fischbach (1978) Neurotransmitters decrease the calcium component of sensory neurone action potentials. Nature 276: 837-839.

Ewald, D., and R. Eckert (1983) Cyclic AMP enhances calcium-dependent potassium current in Aplysia neurons. Cell. Mol. Neurobiol. 3: 340-353.

Ewald, D. A., A. Williams, and I. B. Levitan (1985) Modulation of single $\mathrm{Ca}^{2+}$-dependent $\mathrm{K}+$-channel activity by protein phosphorylation. Nature 315: 503-506.

Gillette, R., M. U. Gillette and W. J. Davis (1982) Substrates of command ability in a buccal neuron of Pleurobranchaea. II. Potential role of cyclic AMP. J. Comp. Physiol. 146: 461-470.

Gilman, A. G. (1984) Guanine nucleotide-binding regulatory proteins and dual control of adenylate cyclase. J. Clin. Invest. 73: 1-4.

Green, D. J., and R. Gillette (1983) Patch- and voltage-clamp analysis of cyclic AMP-stimulated inward current underlying neurone bursting. Nature 306: 784-785.

Grega, D. S., and R. L. Macdonald (1985) Activation of adenylate cyclase in cultured dorsal root ganglion neurons: Involvement of potassium conductance. Neurosci. Abstr. 11: 1093.

Heyer, E. J., and R. L. Macdonald (1982) Calcium- and sodium- 
dependent action potentials of mouse spinal cord and dorsal root ganglion neurons in cell culture. J. Neurophysiol. 47: 641-655.

Huganir, R. L., and P. Greengard (1983) cAMP-dependent protein kinase phosphorylates the nicotinic acetylcholine receptor. Proc. Natl. Acad. Sci. USA 80: 1130-1134.

Kaczmarek, L. K., and F. Strumwasser (1984) A voltage clamp analysis of currents underlying cyclic AMP-induced membrane modulation in isolated peptidergic neurons of Aplysia. J. Neurophysiol. 52 . 340-349.

Kandel, E. R., and J. H. Schwartz (1982) Molecular biology of learning: Modulation of transmitter release by cAMP-dependent protein kinase. Science 218: 433-443.

Kelly, L. A., M. S. Hall, and R. W. Butcher (1974) Cyclic adenosine $3^{\prime}, 5^{\prime}$-monophosphate metabolism in normal and SY40-transformed WI-38 cells. J. Biol. Chem. 249: 5182-5187.

Kupfermann, I. (1980) Role of cyclic nucleotides in excitable cells. Annu. Rev. Physiol. 42: 629-641.

Levitan, I. B. (1985) Phosphorylation of ion channels. J. Membr. Biol. 87: $177-190$.

Levitan, I. B., and W. B. Adams (1981) Cyclic AMP modulation of a specific ion channel in an identified nerve cell: Possible role for protein phosphorylation. Adv. Cyclic Nucleotide Res. 14: 647-653.

Limbird, L. E. (1981) Activation and attenuation of adenylate cyclase. Biochem. J. 195: 1-13.

Macdonald, R. L., J. H. Skerritt, and M. A. Werz (1986) Adenosine agonists reduce voltage-dependent calcium conductance of mouse sensory neurones in culture. J. Physiol. (Lond) 370: 75-90.

Mo, N., R. Ammari, and N. J. Dun (1985) Prostaglandin E $E_{1}$ inhibits calcium-dependent potentials in mammalian sympathetic neurons. Brain Res. 334: 325-329.

Nestler, E. J., and P. Greengard (1983) Protein phosphorylation in the brain. Nature 305: 583-588.

Nowycky, M. C., A. P. Fox, and R. W. Tsien (1985) Three types of neuronal calcium channel with different calcium agonist sensitivity (Letter). Nature 316: 440-443.

Pellman, T. C., and D. O. Carpenter (1981) Cyclic AMP induces a voltage-dependent current in neurons of Aplysia californica. Neurosci. Lett. 22: 151-157.

Ransom, B. R., E. Neale, M. Henkart, P. N. Bullock, and P. G. Nelson (1977) Mouse spinal cord cells in culture. I. Morphologic and intrinsic neuronal electrophysiological properties. J. Neurophysiol. 40: 1132-1150.

Reuter, H. (1983) Calcium channel modulation by neurotransmitters, enzymes and drugs. Nature 301 : 569-574.
Ross, E. M., and A. G. Gilman (1980) Biochemical properties of hormone-sensitive adenylate cyclase. Annu. Rev. Biochem. 49: 533564.

Seamon, K. B., and J. W. Daly (1981) Forskolin: A unique diterpene activator of cyclic AMP-generating systems. J. Cyclic Nucleotide Res. 7: 201-224.

Seelig, T. L., and J. J. Kendig (1982) Cyclic nucleotide modulation of $\mathrm{Na}^{+}$and $\mathrm{K}^{+}$currents in the isolated node of Ranvier. Brain Res. 245: 144-147.

Siegelbaum, S. A., J. S. Camardo, and E. R. Kandel (1982) Serotonin and cAMP close single $\mathrm{K}^{+}$channels in sensory neurones. Nature 299. 413-417.

Solntseva, E. I., and L. V. Bezrukova (1985) Intracellular injection of cAMP and cGMP into snail neurones induces an increase in $\mathrm{Na}^{+}$conductance. Experientia 41: 252-254.

Strong, J. A. (1984) Modulation of potassium current kinetics in bag cell neurons of Aplysia by an activator of adenylate cyclase. J. Neurosci. 4: 2772-2783.

Swandulla, D., and H. D. Lux (1984) Changes in ionic conductances induced by cAMP in Helix neurones. Brain Res. 305: 115-122.

Tsunoo, A., and T. Narahashi (1984) Cyclic AMP-mediated potentiation of muscarinic hyperpolarization in neuroblastoma cells. Brain Res. 294: 123-126.

Van Renterghem, C., J. Penit-Soria, and J. Stinnakre (1985) $\beta$-Adrenergic induced $\mathrm{K}^{+}$current in Xenopus oocytes: Role of cAMP inhibition by muscarinic agents. Proc. R. Soc. London [Biol.] 223:389402.

Walsh, J. P., and J. H. Byrne (1984) Forskolin mimics and blocks a serotonin-sensitive decreased $\mathrm{K}^{+}$conductance in tail sensory neurons of Aplysia. Neurosci. Lett. 52: 7-11.

Werz, M. A., and R. L. Macdonald (1983) Opioid peptides with differential affinity for mu and delta receptors decrease sensory neuron calcium-dependent action potentials. J. Pharmacol. Exp. Ther. 227 394-402.

Werz, M. A., and R. L. Macdonald (1985) Dynorphin and neoendorphin peptides decrease dorsal root ganglion neuron calcium-dependent action potential duration. J. Pharmacol. Exp. Ther. 234: 4956.

Wiener, E. C., and W. R. Loewenstein (1983) Correction of cell-cell communication defect by introduction of a protein kinase into mutant cells. Nature 305: 433-435. 